\title{
Características endoscópicas del linfoma gástrico primario
}

\author{
Ricardo Balanzá1*, Nicole Somerville², Fernando Rojas-Mendoza ${ }^{1}$ y Nancy E. Aguilar-Olivos ${ }^{1}$

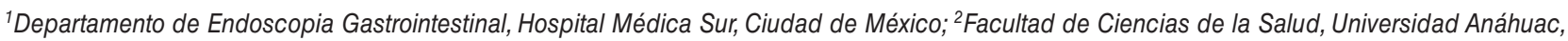 \\ Estado de México. México
}

\begin{abstract}
Resumen
Introducción y objetivo: El linfoma gástrico primario representa el 5\% de las neoplasias gástricas. El diagnóstico se realiza principalmente por esofagogastroduodenoscopia. No existen lesiones patognomónicas, ya que puede presentarse con alteraciones superficiales de la mucosa, protrusión, infiltración difusa, ulceración, engrosamiento de pliegues gástricos e incluso en mucosa aparentemente normal. El objetivo de este estudio es realizar una revisión de casos de linfoma gástrico primario para describir y reportar las características endoscópicas, morfología y localización, más frecuentes en esta patología. Material y métodos: Se llevó a cabo una búsqueda de casos de linfoma gástrico primario en la base de datos de Anatomía Patológica de nuestro hospital de enero de 2015 a enero de 2020. Se analizaron las características endoscópicas de los pacientes diagnosticados por esofagogastroduodenoscopia. Resultados: Se analizaron 13 casos de linfoma gástrico primario. Los pliegues gástricos se encontraban engrosados en todos los pacientes y ninguno presentó mucosa aparentemente normal. Ocho pacientes (61.54\%) presentaron úlceras, 6 (46.15\%) alteraciones superficiales de la mucosa, $3(23.08 \%)$ lesiones elevadas y 2 (15.38\%) pseudopólipos. La localización fue $13(100.00 \%)$ en cuerpo, 7 (53.85\%) en fondo y cardias, $3(23.08 \%)$ en antro, $11(84.62 \%)$ lesiones extensas y 2 (15.38\%) lesiones focales. Conclusiones: El diagnóstico de linfoma gástrico primario se realiza principalmente por medio de esofagogastroduodenoscopia, por lo que es de suma importancia conocer sus características endoscópicas. En nuestra serie de casos las características endoscópicas más frecuentemente asociadas a linfoma gástrico primario fueron engrosamiento de pliegues gástricos y localización en cuerpo.
\end{abstract}

Palabras clave: Linfoma gástrico primario. Esofagogastroduodenoscopia. Características endoscópicas. Engrosamiento de pliegues gástricos. Cáncer.

\section{Endoscopic characteristics of primary gastric lymphoma}

\section{Abstract}

Introduction and objective: Primary gastric lymphoma accounts for 5\% of all gastric neoplasia. Esophagogastroduodenoscopy is frequently the diagnostic method of choice, although no pathognomonic lesions have been described since it may manifest as superficial alterations of the mucosa, protrusion, diffuse infiltration, ulceration, thickened gastric folds, and even as apparently normal mucosa. The objective of this study is to evaluate a series of cases of primary gastric lymphoma in order to describe and report the most frequent endoscopic characteristics, morphology and localization, of this pathology. Material and methods: We searched for all the primary gastric lymphoma cases in the Anatomic Pathology database at our hospital from January 2015 to January 2020. The endoscopic characteristics of the patients diagnosed by esophagogastro-

Correspondencia:

*Ricardo Balanzá

E-mail: balanza.ricardo@gmail.com
Fecha de recepción: 11-02-2020

Fecha de aceptación: 27-03-2020

DOI: 10.24875/END.20000013
Disponible en internet: 08-05-2020

Endoscopia. 2020;32(1):7-13 www.endoscopia-ameg.com 
duodenoscopy were analyzed. Results: We analyzed 13 cases of primary gastric lymphoma. Thickened gastric folds were observed in all patients and none had apparently normal mucosa. Eight patients (61.54\%) presented ulcers, 6 (46.15\%) superficial mucosal alterations, $3(23.08 \%)$ elevated lesions, and $2(15.38 \%)$ pseudopolyps. Thirteen cases (100\%) were located in the body of the stomach, $7(53.85 \%)$ in the cardias and fundus, $3(23.08 \%)$ in the antrum, $11(84.62 \%)$ were extended lesions, and $2(15.38 \%)$ were focal lesions. Conclusions: The diagnosis of primary gastric lymphoma is usually performed by esophagogastroduodenoscopy, resulting crucial to recognize and describe its endoscopic characteristics. In our case series the most frequently associated endoscopic characteristics to primary gastric lymphoma were the presence of thickened gastric folds and the involvement of the gastric body.

Key words: Primary gastric lymphoma. Esophagogastroduodenoscopy. Endoscopic characteristics. Thickened gastric folds. Cancer.

\section{Introducción}

El linfoma gástrico primario es un tumor raro, ya que tan solo representa el $5 \%$ de las neoplasias gástricas primarias $^{1-3}$. La frecuencia de linfoma gástrico primario en México ha aumentado del 1 al $1.9 \%$ de todas las neoplasias gástricas en 1960 hasta el $9.1 \%$ en el $2001^{4}$. El estómago es la localización más común de presentación para los linfomas extranodales y representa del 4 al $20 \%$ de estos ${ }^{1,2}$. El 55 al $65 \%$ de los linfomas gastrointestinales se localizan en el estómago $0^{1,5}$.

La Organización Mundial de la Salud clasifica los linfomas gástricos primarios de acuerdo con su histología en linfoma difuso de células B grandes y linfoma de tejido linfoide asociado a mucosa (MALT, por las siglas en inglés de mucosa-associated lymphoid tis$s u e)^{6}$. El linfoma difuso de células B grandes es una neoplasia de alto grado y el tipo histológico más frecuente de linfoma gástrico primario'. Los linfomas de tejido linfoide asociado a mucosas son neoplasias de bajo grado usualmente secundarias a infección por Helicobacter pylori ${ }^{1,7}$.

La exploración física en casos de linfoma gástrico primario puede ser normal hasta en el $55-60 \%$ de los casos $^{8}$. Los síntomas más comunes al momento de diagnóstico son dolor epigástrico (78-80.6\%), anorexia $(47 \%)$, pérdida de peso no intencional (17.8-25\%), hemorragia de tubo digestivo (19-36.8\%) y vómito $(18 \%)^{1,9,10}$. Los síntomas B solo se presentan en el $12 \%$ de los $\operatorname{casos}^{9,10}$.

El diagnóstico de linfoma gástrico primario se realiza por medio de la toma de biopsias gástricas durante una esofagogastroduodenoscopia ${ }^{9}$. La presentación endoscópica de linfoma gástrico primario se caracteriza por una variedad de patrones no específicos que pueden sugerir malignidad, como úlceras múltiples o gigantes y lesiones elevadas, así como la presencia de enfermedad benigna, ya sean erosiones, nódulos pequeños, engrosamiento de pliegues gástricos, eritema o mucosa aparentemente normal ${ }^{11}$. La mayoría de los linfomas gástricos primarios se localizan en el antro, seguidos por el cuerpo y fondo' ${ }^{12}$.

Históricamente, el tratamiento para linfoma gástrico primario se basaba en la resección quirúrgica, sin embargo actualmente se ha demostrado que esta estrategia no confiere un beneficio en la supervivencia ${ }^{13}$. El tratamiento de erradicación de $H$. pylori se considera el tratamiento inicial en pacientes con diagnóstico de MALT localizado con evidencia de infección por dicha bacteria $^{14}$. La radioterapia se recomienda en pacientes sin respuesta a tratamiento para erradicación de $\mathrm{H}$. pylori, en caso de recidiva o en pacientes con la translocación $t(11 ; 18)^{9}$. El tratamiento para linfoma difuso de células $B$ grandes se basa en quimioterapia e inmunoterapia, independientemente del estadio de la enfermedad ${ }^{15}$. El tratamiento quirúrgico en cualquier tipo de linfoma gástrico primario se encuentra reservado para complicaciones como sangrado, perforación u obstrucción?

Es necesario conocer la posible morfología y localización de esta neoplasia para la toma de biopsias y consiguiente diagnóstico oportuno. El objetivo de este estudio es realizar una revisión de casos de linfoma gástrico primario para describir y reportar las características endoscópicas, morfología y localización más frecuentes en esta patología.

\section{Material y métodos}

Se llevó a cabo una búsqueda de casos de linfoma gástrico primario en la base de datos del Servicio de Anatomía Patológica de nuestro hospital de enero de 2015 a enero de 2020. Posteriormente se identificaron los casos diagnosticados por esofagogastroduodenoscopia en el mismo hospital y se evaluaron las imágenes y reportes endoscópicos. Todos los estudios endoscópicos se realizaron con equipos Olympus GIF-H180 y Olympus GIF-HQ190. Los criterios de inclusión fueron pacientes mayores de 18 años con diagnóstico de 
linfoma gástrico primario, diagnóstico realizado con base en toma de biopsias por esofagogastroduodenoscopia, imágenes, reportes endoscópicos y de patología disponibles. Los criterios de exclusión abarcaron diagnóstico previo a esofagogastroduodenoscopia, resección gástrica, linfoma gástrico secundario, enfermedad inflamatoria intestinal, enfermedad celiaca y síndromes asociados a pólipos. Se analizaron las características endoscópicas, morfología y localización, de los casos que cumplieran los criterios de inclusión. Las imágenes endoscópicas fueron revisadas por dos gastroenterólogos y endoscopistas del aparato digestivo certificados por el Consejo Mexicano de Gastroenterología para determinar las características endoscópicas de cada caso.

\section{Resultados}

Se identificaron 15 casos de linfoma gástrico primario en la base de datos del Servicio de Anatomía Patológica de nuestro hospital. En 14 pacientes el diagnóstico se realizó por esofagogastroduodenoscopia y 13 pacientes cumplieron los criterios de inclusión para el presente estudio. Se analizaron las características endoscópicas, morfología y localización de 13 casos de linfoma gástrico primario.

El grupo de pacientes incluidos en este estudio consistió en 9 (69.23\%) hombres y $4(30.77 \%)$ mujeres. La edad promedio de los pacientes fue de 64.69 años, con un intervalo de 37 a 85 años. El tipo histológico más frecuente fue linfoma difuso de células $B$ grandes con 8 (61.54\%) casos, seguido de MALT con 5 (38.46\%) casos. Se documentó infección por $H$. pylori en $3(23.08 \%)$ casos, el $60.00 \%$ de los pacientes con diagnóstico de MALT y un $0.00 \%$ de los pacientes con diagnóstico de linfoma difuso de células $\mathrm{B}$ grandes (Tabla 1).

En cuanto a la morfología, $13(100 \%)$ pacientes presentaron pliegues gástricos engrosados, 8 (61.54\%) presentaron úlceras, $6(46.15 \%)$ alteraciones superficiales de la mucosa como eritema o erosiones, $3(23.08 \%)$ lesiones elevadas, 2 (15.38\%) pseudopólipos y 0 (0.00\%) mucosa aparentemente normal (Fig. 1). La localización de los linfomas gástricos primarios identificados fue $13(100.00 \%)$ en cuerpo, 7 (53.85\%) en fondo y cardias, y $3(23.08 \%)$ en antro. Respecto a la extensión de las lesiones, $11(84.62 \%)$ pacientes presentaron lesiones extensas y 2 (15.38\%) lesiones focales (Fig. 2).

\section{Discusión}

El linfoma gástrico primario representa el $5 \%$ del total de las neoplasias gástricas, por lo cual se considera
Tabla 1. Características de los pacientes con diagnóstico de linfoma gástrico primario.

\begin{tabular}{|l|c|c|}
\hline Edad & \multicolumn{2}{|c|}{ Años } \\
\hline Media & \multicolumn{2}{|c|}{64.69} \\
\hline Mediana & \multicolumn{2}{|c|}{63} \\
\hline Intervalo mínimo-máximo & \multicolumn{2}{|c|}{$37-85$} \\
\hline Sexo & Número & Porcentaje \\
\hline Hombres & 9 & $69.23 \%$ \\
\hline Mujeres & 4 & $30.77 \%$ \\
\hline Tipo histológico & & \\
\hline Linfoma difuso de células B grandes & 8 & $61.54 \%$ \\
\hline MALT & 5 & $38.46 \%$ \\
\hline Infección por $\boldsymbol{H}$. pylori & & \\
\hline Total & 3 & $23.08 \%$ \\
\hline MALT & 3 & $60.00 \%$ \\
\hline Linfoma difuso de células B grandes & 0 & $00.00 \%$ \\
\hline
\end{tabular}

MALT: linfoma de tejido linfoide asociado a mucosa.

un tumor raro ${ }^{1,2}$. El estómago es el sitio más común de presentación para linfomas no Hodgkin extranodales y representa del 30 al $40 \%$ de estos ${ }^{1}$. El estómago es el principal órgano afectado dentro de los linfomas gastrointestinales primarios, con un estimado del 55 al $65 \%$ de los mismos, seguido por el intestino delgado, la región ileocecal y el recto ${ }^{16,17}$. La incidencia de linfoma gástrico primario ha incrementado de manera constante en las últimas décadas de acuerdo con datos reportados en el Programa de Vigilancia, Epidemiología y Resultados Finales del Instituto Nacional de Cáncer de EE.UU ${ }^{18}$. En México se ha observado un incremento similar en la incidencia de linfoma gástrico primario, ya que en 1960 esta patología representaba del 1 al $1.9 \%$ de todas las neoplasias gástricas, lo cual incrementó al $9.1 \%$ en el año $2001^{4}$.

En nuestra serie, el $69.23 \%$ de los pacientes son hombres y el $30.77 \%$ mujeres, lo cual concuerda con la literatura mundial, en donde se reporta una relación de 2 a 3 hombres por una mujer ${ }^{3,8,16}$. La edad promedio de diagnóstico en nuestra serie es de 64.69 años, por lo que coincide con la edad documentada a nivel mundial, la cual corresponde a mayores de 50 años ${ }^{3,8,16}$.

La distribución de los principales tipos histológicos de linfoma gástrico primario por orden de frecuencia en Alemania es linfoma difuso de células B grandes (59\%), 


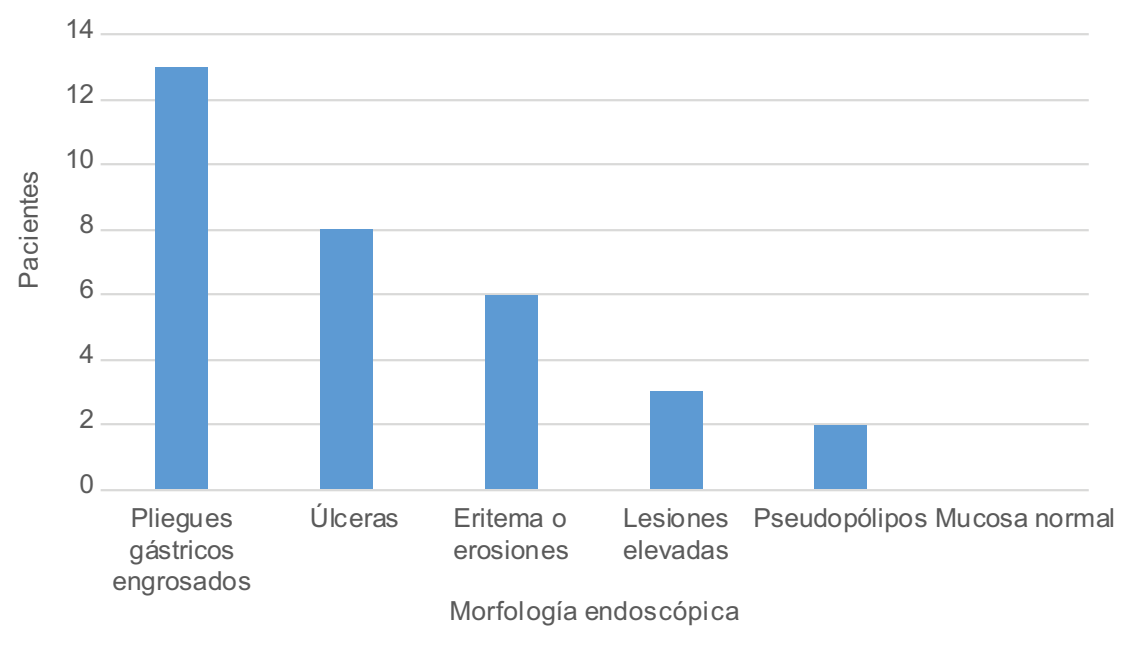

Figura 1. Morfología endoscópica de linfoma gástrico primario en nuestra serie.

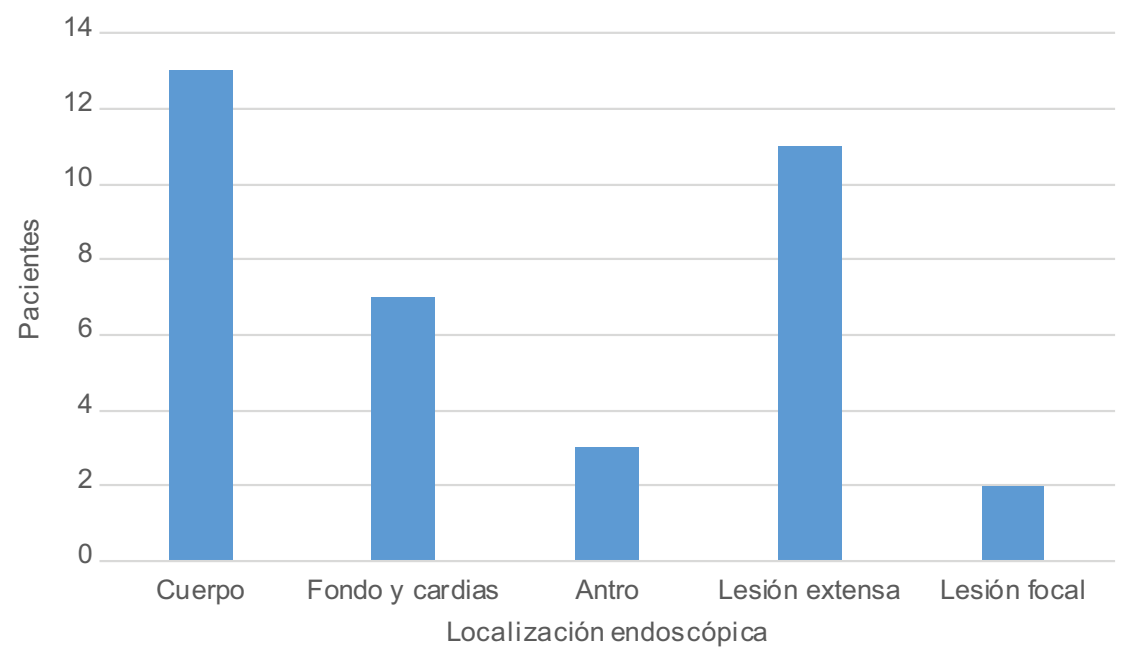

Figura 2. Localización endoscópica de linfoma gástrico primario en nuestra serie.

MALT (38\%), linfoma de células T periférico (1.5\%), linfoma de células del manto (1\%) y linfoma folicular $(0.5 \%)^{7}$. En China la proporción reportada de tipos histológicos de linfoma gástrico primario es del $65.5 \%$ para linfoma difuso de células B grandes y del $31.5 \%$ para MALT ${ }^{1}$. La distribución en India es del $62.8 \%$ para linfoma difuso de células $B$ grandes, el $34.8 \%$ para MALT y el $2.3 \%$ para linfoma folicular ${ }^{3}$. Nuestra serie de casos muestra una tendencia similar a la reportada en estos tres países, con una distribución de tipos histológicos del $61.54 \%$ para linfoma difuso de células B grandes, siendo el más frecuente, y el $38.46 \%$ para MALT.
El estímulo antigénico producido secundario a la infección crónica por $H$. pylori favorece la expansión clonal de células linfoides característica del MALT ${ }^{19}$. El porcentaje de pacientes con diagnóstico de linfoma gástrico primario e infección por $H$. pylori varía dependiendo de la serie desde un 41 hasta un $85 \%^{3,20,21}$. El MALT se asocia a infección por $H$. pylori en el 78 al $80.3 \%$ de los $\operatorname{casos}^{21}$. En nuestra serie, el porcentaje de pacientes con infección por $\mathrm{H}$. pylori documentada histológicamente es el $23.08 \%$ sin embargo, tomando en cuenta solo los pacientes con diagnóstico de MALT, el porcentaje asciende al $60.00 \%$. 


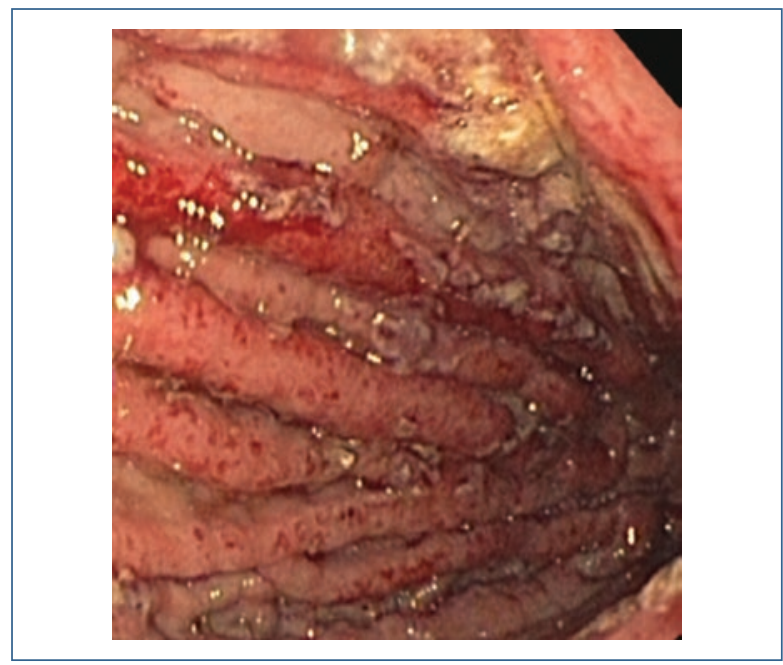

Figura 3. Pliegues gástricos engrosados secundarios a linfoma gástrico primario en hombre de 37 años de edad.

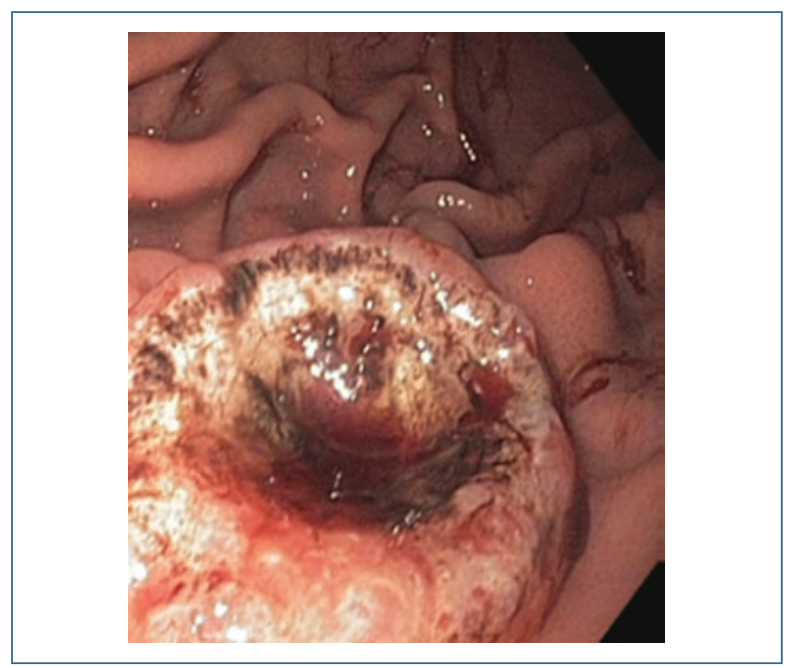

Figura 4. Úlcera secundaria a linfoma gástrico primario en hombre de 55 años de edad.

El estándar de oro para el diagnóstico de linfoma gástrico primario es la toma de biopsias por medio de esofagogastroduodenoscopia ${ }^{8}$. La esofagogastroduodenoscopia no puede discernir por sí sola entre la presencia de linfoma gástrico primario, adenocarcinoma gástrico o patología benigna, sin embargo puede identificar patrones sugestivos de la enfermedad, como ulceración, infiltración difusa o lesiones elevadas ${ }^{8}$. Es necesario tomar múltiples biopsias profundas, de preferencia por medio de resección endoscópica de la mucosa, tanto de mucosa anormal como normal para incrementar la exactitud diagnóstica debido a que el

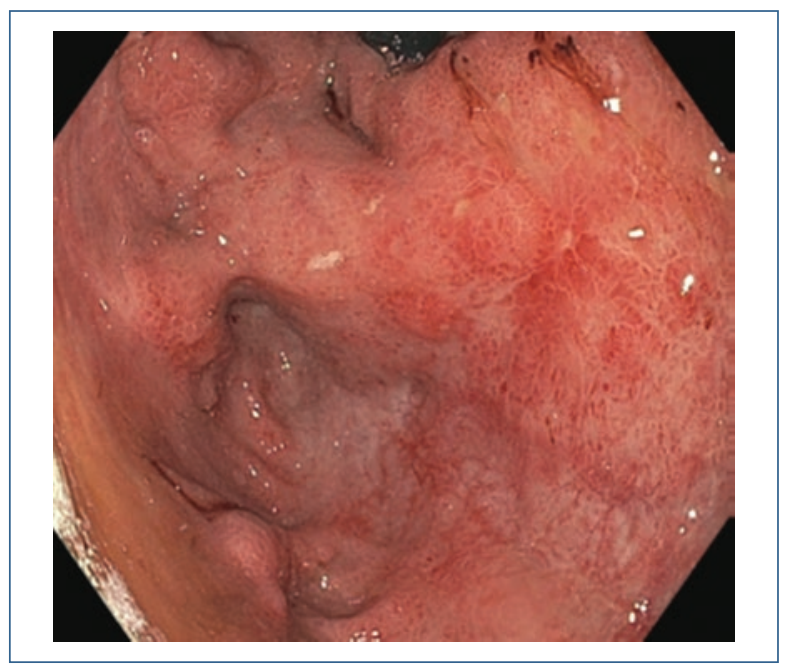

Figura 5. Eritema y erosiones secundarias a linfoma gástrico primario en hombre de 64 años de edad.

tumor puede ser multifocal o infiltrar la capa submucosa debajo de mucosa aparentemente normal ${ }^{8}$. Hasta un tercio de los linfomas gástricos primarios pueden tener una apariencia endoscópica benigna ${ }^{12}$. El ultrasonido endoscópico es una técnica útil para determinar la profundidad de la lesión y la presencia de nódulos perigástricos afectados ${ }^{8}$.

La morfología endoscópica de linfoma gástrico primario reportada en una serie de 144 casos en Italia fue, en orden de frecuencia, presencia de úlceras $(60.00 \%)$, mucosa aparentemente normal $(10.00 \%)$, lesiones elevadas $(9.72 \%)$, eritema $(5.00 \%)$, lesiones nodulares (4.86\%), pliegues gástricos engrosados (4.86\%) y pseudopólipos $(0.69 \%)^{11}$. En India, la morfología endoscópica reportada en una serie de 43 casos fue presencia de pseudopólipos en el $44.18 \%$ de los casos, pliegues gástricos engrosados en el $27.91 \%$ de los casos, úlceras en el $23.25 \%$ y erosiones en el $4.65 \%{ }^{3}$. En nuestra serie, el $100.00 \%$ de los pacientes presentó pliegues gástricos engrosados (Fig. 3), el 61.54\% úlceras gástricas (Fig. 4), el $46.15 \%$ lesiones superficiales de la mucosa como eritema o erosiones (Fig. 5), el $23.08 \%$ lesiones elevadas (Fig. 6), el 15.38\% pseudopólipos (Fig. 7) y ninguno mucosa aparentemente normal. No existen imágenes endoscópicas patognomónicas para el diagnóstico de linfoma gástrico primario y de acuerdo con lo antes mencionado, la morfología es sumamente variable. En nuestra serie, la morfología más frecuentemente identificada fue la presencia de pliegues gástricos engrosados, mientras que en series de Italia e India solo representa el 4.86 y el $27.91 \%$ 


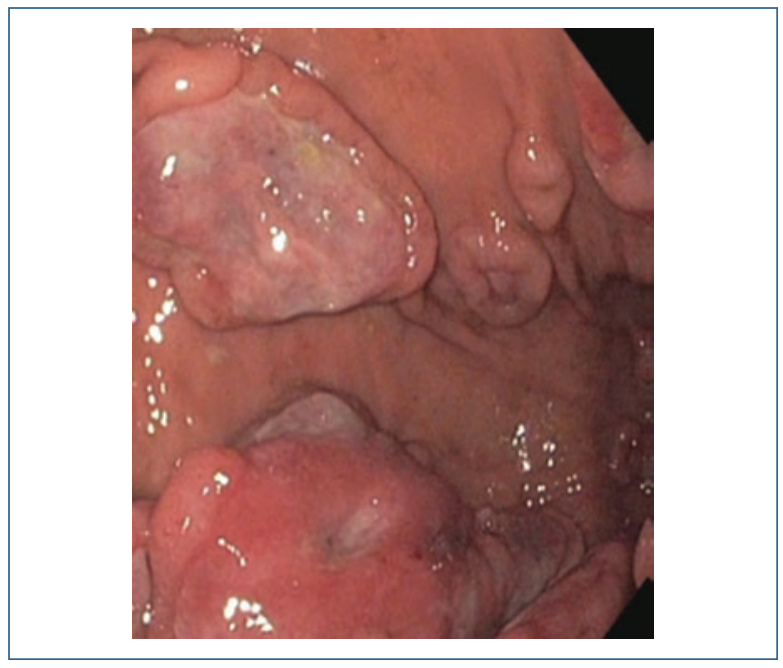

Figura 6. Lesiones elevadas secundarias a linfoma gástrico primario en mujer de 61 años de edad.

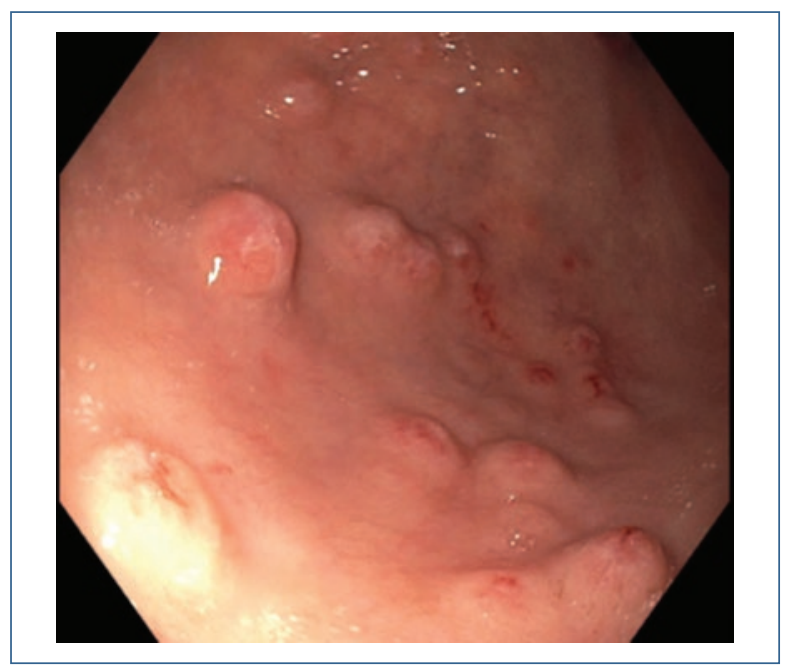

Figura 7. Pseudopólipos secundarios a linfoma gástrico primario en hombre de 61 años de edad.

respectivamente. El diagnóstico de linfoma gástrico primario en mucosa aparentemente normal se ha descrito hasta en el $10 \%$ de los casos, sin embargo en nuestra serie ninguno de los casos diagnosticados cuenta con esta característica.

El linfoma gástrico primario se localiza de manera frecuente en antro (50\%) y en menor proporción en cuerpo (24\%) y fondo ${ }^{12}$. En Europa se ha reportado que el linfoma gástrico primario afecta al antro en el $37 \%$ de los casos, el cuerpo y fondo en el $44 \%$ de los casos y se presenta como una lesión extensa en el $18 \%$ de los ca$\operatorname{sos}^{11}$. En una serie de 43 pacientes en India la
Tabla 2. Diferencias en las características endoscópicas de linfoma gástrico primario reportadas en la literatura mundial y nuestra serie.

\begin{tabular}{|l|c|c|}
\hline Características endoscópicas & $\begin{array}{c}\text { Serie } \\
\text { reportada }\end{array}$ & $\begin{array}{c}\text { Literatura } \\
\text { mundial }\end{array}$ \\
\hline Pliegues gástricos engrosados & $100.00 \%$ & $4.86-27.91 \%$ \\
\hline Úlceras & $61.54 \%$ & $23.25-60.00 \%$ \\
\hline Eritema o erosiones & $46.15 \%$ & $4.65-5.00 \%$ \\
\hline Lesiones elevadas & $23.08 \%$ & $9.72 \%$ \\
\hline Pseudopólipos & $15.38 \%$ & $0.69 \%-44.18 \%$ \\
\hline Mucosa normal & $0.00 \%$ & $10.00 \%$ \\
\hline Cuerpo & $100.00 \%$ & $24.00-27.91 \%$ \\
\hline Fondo y cardias & $53.85 \%$ & $4.65 \%$ \\
\hline Antro & $23.08 \%$ & $37.00-50.00 \%$ \\
\hline Lesión extensa & $84.62 \%$ & $18.00-20.93 \%$ \\
\hline Lesión focal & $15.38 \%$ & $82.00-79.07 \%$ \\
\hline
\end{tabular}

localización fue el $46.51 \%$ en antro, el $27.91 \%$ en cuerpo, el $4.65 \%$ en fondo y el $20.93 \%$ como lesiones extensas ${ }^{3}$. En nuestra serie de casos, la localización de esta neoplasia fue cuerpo en el $100.00 \%$ de los casos, fondo y cardias en el $53.85 \%$ de los casos y antro en el $23.08 \%$. Los linfomas gástricos primarios identificados se presentaron como lesiones extensas en el $84.62 \%$ de los casos y como lesiones focales en el $15.38 \%$. La localización principal de esta patología reportada en la literatura difiere de nuestra serie, ya que mientras el $100 \%$ de nuestros casos tuvieron involucro del cuerpo gástrico, tan solo del 24 al $28 \%$ de los casos reportados a nivel mundial presentan esta característica. El antro es el segmento gástrico principalmente afectado en la patogénesis del linfoma gástrico primario de acuerdo con la literatura, sin embargo en nuestra serie representa tan solo el $23.08 \%$ de los casos. Las diferencias en las características endoscópicas reportadas en la literatura mundial y nuestra serie se encuentran resumidas en la tabla 2.

\section{Conclusiones}

La esofagogastroduodenoscopia con toma de biopsias de mucosa gástrica es el estándar de oro para el diagnóstico de linfoma gástrico primario. Las características endoscópicas, morfología y localización, de esta patología son diversas y no existe una lesión patognomónica para su diagnóstico. Es de suma importancia conocer las múltiples presentaciones 
endoscópicas posibles del linfoma gástrico primario para obtener tejido, realizar el análisis histopatológico y así lograr un diagnóstico correcto y oportuno.

La edad promedio de presentación para linfoma gástrico primario en los pacientes incluidos en el presente estudio es de 64.69 años, con una marcada predilección por el sexo masculino, ya que el $69.23 \%$ de los pacientes son hombres. El tipo histológico más frecuentemente identificado en nuestro hospital es el linfoma difuso de células B grandes, reportado en el $61.54 \%$ de los casos. La edad de presentación, la predilección por el sexo masculino y la distribución de los tipos histológicos de linfoma gástrico primario reportada por nuestro grupo coincide con lo mostrado en la literatura mundial.

En nuestra serie de casos, la morfología endoscópica más frecuentemente identificada fue el engrosamiento de los pliegues gástricos, lo cual difiere de la literatura mundial con un porcentaje del $100.00 \%$ frente al 4.86 a $27.91 \%$. El hallazgo de linfoma gástrico primario en mucosa aparentemente normal se encuentra descrito y conforma hasta el $10 \%$ de los casos de este tipo de cáncer, sin embargo en nuestra serie de casos no se identificó dicha característica endoscópica. La localización endoscópica principal en nuestra serie de casos es el cuerpo gástrico (100.00\%), mientras que la literatura mundial menciona una predilección por el antro $(50.00 \%)$. Es necesario un mayor número de pacientes para confirmar las diferencias antes mencionadas en la presentación endoscópica de linfoma gástrico primario en pacientes mexicanos.

\section{Financiamiento}

Los autores no recibieron patrocinio para llevar a cabo este artículo.

\section{Conflicto de intereses}

Los autores declaran no tener conflicto de intereses alguno.

\section{Responsabilidades éticas}

Protección de personas y animales. Los autores declaran que para esta investigación no se han realizado experimentos en seres humanos ni en animales.

Confidencialidad de los datos. Los autores declaran que han seguido los protocolos de su centro de trabajo sobre la publicación de datos de pacientes.
Derecho a la privacidad y consentimiento informado. Los autores han obtenido el consentimiento informado de los pacientes y/o sujetos referidos en el artículo. Este documento obra en poder del autor de correspondencia.

\section{Bibliografía}

1. Wang YG, Zhao LY, Liu CQ, Pan SC, Chen XL, Liu K, et al. Clinical characteristics and prognostic factors of primary gastric lymphoma. A retrospective study with 165 cases. Medicine (Baltimore). 2016;95(31):e4250.

2. Al-Akwaa AM, Al-Mofleh SIA. Primary gastric lymphoma. World J Gastroenterol. 2004;10(1):5-11

3. Malipatel R, Patil M, Rout $P$, Correa M, Devarbhavi H. Primary gastric lymphoma: Clinicopathological profile. Euroasian J Hepatogastroenterol. 2018;8(1):6-10.

4. Arista J, Jiménez F, Noble A, Lazos M, Cuesta T, Cortés E, et al. Frecuencia de linfoma gástrico en seis hospitales de la Ciudad de México. Rev Gastroenterol Mex. 2001;66:96-100.

5. Ghimire P, Wu GY, Zhu L. Primary gastrointestinal lymphoma. World J Gastroenterol. 2011;17(6):697-707.

6. Doglioni C, Ponzoni M, Ferreri AJM, Savio A; Gruppo Italiano Patologi Apparato Digerente (GIPAD); Società Italiana di Anatomia Patologica e Citopatologia Diagnostica/International Academy of Pathology, Italian division (SIAPEC/IAP). Gastric lymphoma: the histology report. Dig Liver Dis. 2011;43:S310-8.

7. Koch P, Probst A, Berdel WE, Willich NA, Reinartz G, Brockmann J, et al. Treatment results in localized primary gastric lymphoma: data of patients registered within the German multicenter study (GIT NHL 02/96). J Clin Oncol. 2005;23:7050-9.

8. Juárez-Salcedo LM, Sokol L, Chavez JC, Dalia S. Primary gastric lymphoma, epidemiology, clinical diagnosis, and treatment. Cancer Control. 2018:25:1-12.

9. Ikoma N, Badgwell BD, Mansfield PF. Multimodality treatment of gastric lymphoma. Surg Clin N Am. 2016;97(2):405-20.

10. Koch $P$, del Valle F, Berdel WE, Willich NA, Reers B, Hiddemann W, et al.; German Multicenter Study Group. Primary gastrointestinal non-Hodgkin's lymphoma: I. Anatomic and histologic distribution, clinical features, and survival data of 371 patients registered in the German Multicenter Study GIT NHL 01/92. J Clin Oncol. 2001;19(18):3861-73.

11. Andriani A, Zullo A, Di Raimondo F, Patti C, Tedeschi L, Recine U, et al. Clinical and endoscopic presentation of primary gastric lymphoma: a multicentre study. Aliment Pharmacol Ther. 2006;23:721-6.

12. Chang MC, Kuo SH. Primary gastric lymphoma. J Gastroenterol. 2017;3(1):1006.

13. Binn M, Ruskone-Fourmestraux A, Lepage E, Haioun C, Delmer A, Aegerter $\mathrm{P}$, et al. Surgical resection plus chemotherapy versus chemotherapy alone: comparison of two strategies to treat diffuse large B-cell gastric lymphoma. Ann Oncol. 2003;13(12):1751-7.

14. Zucca E, Copie-Bergman C, Ricardi U, Thieblemont C, Raderer M, Ladetto M; ESMO Guidelines Working Group. Gastric marginal zone lymphoma of MALT type: ESMO clinical practice guidelines for diagnosis, treatment and follow-up. Ann Oncol. 2013;24(Suppl 6):vi144-8.

15. Vitolo U, Seymour JF, Martelli M, Illerhaus G, Illidge T, Zucca E, et al.; ESMO Guidelines Committee. Extranodal diffuse large B-cell lymphoma (DLBCL) and primary mediastinal B-cell lymphoma: ESMO Clinical Practice Guidelines for diagnosis, treatment and follow-up. Ann Oncol. 2016;27(Suppl 5):v91-102.

16. Guimire $P$, Wu GY, Zhu L. Primary gastrointestinal lymphoma. World J Gastroenterol. 2011;17:687-707.

17. Papaxoinis G, Papageorgius S, Rontogianni D, Kaloutsi V, Fountzilas G, Pavlidis N, et al. Primary gastrointestinal non-Hodgkin's lymphoma: a clinicopathologic study of 128 cases in Greece. A Hellenic Cooperative Oncology Group study (HeCOG). Leuk Lymphoma. 2006;47:2140-6.

18. Severson RK, Davis S. Increasing incidence of primary gastric lymphoma. Cancer. 1990;66:1283-7.

19. Moleiro J, Ferreira S, Lage P, Dias Pereira A. Gastric malt lymphoma: Analysis of a series of consecutive patients over 20 years. United European Gastroenterol J. 2016;4(3):395-402.

20. Arora N, Manipadan MT, Pulimood A, Ramakrishna BS, Chacko A, Kurian SS, et al. Gastrointestinal lymphomas: pattern of distribution and histological subtypes: 10 years experience in a tertiary centre in South India. Indian J Pathol Microbiol. 2011;54(4):712-9.

21. Delchier JC, Lamarque D, Levy M, Tkoub EM, Copie-Bergman C, Deforges $\mathrm{L}$, et al. Helicobacter and gastric lymphoma: high prevalence of Cag $A$ in diffuse large $B$ cell lymphoma but not in low grade lymphoma of mucosa-associated lymphoid tissue type. Am J Gastroenterol. 2001;96(8):2324-8. 